\title{
Photoconduction in Intramolecular Charge Transfer Complex Dispersed Polymer Films
}

\author{
Ryo HIROHASHI,* Norihisa KobaYASHI, and Tomohisa SUZUKI \\ Department of Image Science and Engineering, Chiba University, \\ Chiba 260, Japan
}

(Received July 8, 1989)

\begin{abstract}
Azo benzene derivatives are dispersed in a poly(carbonate) or $\operatorname{poly}(N$-vinyl carbazole) matrix, and their photoconductive characteristics are investigated in terms of the structure of azo benzene derivatives and polymer matrix. These azo benzene derivatives are composed of electron donating and accepting parts bridged by azo bonds and are intramolecular charge transfer complexes as determined by UV-Vis spectra and electrochemical measurements. This charge transfer is effective for the increase of photoconduction in these derivative-dispersed poly(carbonate) films. When poly( $N$-vinyl carbazole) is used as a polymer matrix, photocurrent increases by one order of magnitude in comparison with that in a poly(carbonate) system. The roles of azo derivatives and polymers for photoconduction are discussed with the action spectrum of the photocurrent in these systems.

KEY WORDS Photocurrent / Azo Dyes / Intramolecular Charge Transfer / Poly(carbonate) / Poly ( $N$-vinyl carbazole) / Thin Film /
\end{abstract}

The organic photoconductor, or OPC, has attracted much interest for theoretical and basic research as well as for applications to photoreceptors used in electrophotography. Actually, some of these materials have been commercially used in photocopiers, laser printers and electrophotography. Recent development of OPC has been drastically activated since the preparation of poly $(N$-vinyl carbazole) and research on its photoconductive characteristics in the latter half of 1950's. ${ }^{1}$ Especially, sensitization of photoconductivity in poly ( $N$-vinyl carbazole) by adding electron acceptor has been widely reported,,$^{2,3}$ and its carrier generation and transport characteristics have also been investigated. ${ }^{4-6}$ This sensitization is revealed to be due to intermolecular charge transfer interaction between electron donating carbazolyl groups on the polymer and electron accepting molecules. Other numerous polymers containing carbazolyl groups and suitable electron accepting molecules or polymers have been synthesized and investigated in order to realize excellent carrier generation and transport. ${ }^{7-10}$

Photosensitive molecules dispersed polymer films are also investigated as OPC. Triphenylmethane and hydrazone derivatives are typically used as charge transport materials, ${ }^{11-13}$ and these are dispersed in inert matrices such as poly(carbonate) at high concentration. The present authors have already reported the photoconduction mechanism in rhodanine derivatives ${ }^{14}$ and phthalocyanines. ${ }^{15}$ Especially, photoconduction in rhodanine derivatives is revealed to be considerably affected by the effect of electron accepting and donating substituents.

These investigations suggest that the photoconduction in the above mentioned OPCs is considerably affected by interaction between the electron acceptor and donor. The introduc-

\footnotetext{
* To whom all correspondence should be addressed.
} 
tion of this interaction into photosensitive molecules is therefore considered to be useful as one of the molecular designs of OPC. This paper describes photoconduction in polymer films containing azo benzene derivatives which are composed of electron accepting and donating parts bridged by azo bonds. The effects of polymer matrices and intramolecular charge transfer in azo benzene derivatives on photoconduction are also discussed.

\section{EXPERIMENTAL}

\section{Materials}

The structures of azo benzene derivatives, abbieviated as JC dye (JC-7, 8 and 9) are shown in Figure 1. These dyes were obtained from Nippon Kayaku Co., Ltd. The samples were purified by Soxhlet extraction twice with acetone. The acetone soluble part was collected, and its structure was confirmed by elementary analyses, FT-IR (Perkin Elmer Model 1640) and ${ }^{1} \mathrm{H}$ NMR spectra. AC-2 and DO-2 were also obtained from Nippon Kayaku Co., Ltd., and used as model molecules for the accepting and donating parts of JC-9, respectively. Bisphenol-A type poly-
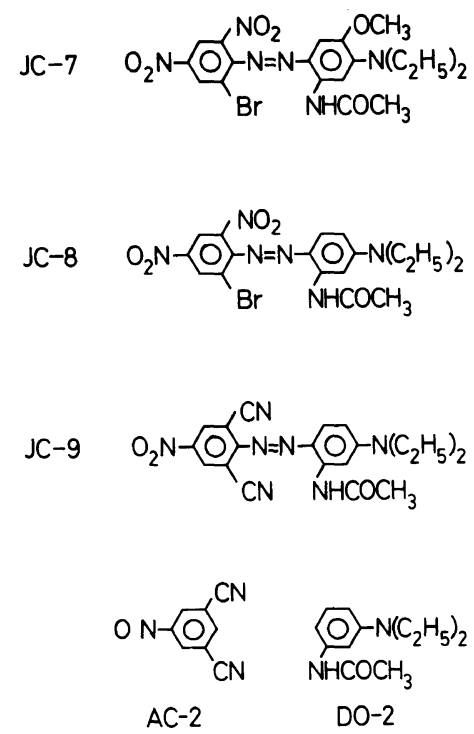

Figure 1. Structures of azo benzene derivatives. (carbonate) (PC) and poly $(N$-vinyl carbazole) $(\mathrm{PVCz})$ were selected as matrix polymers. These polymers were purified by the reprecipitation with methanol for three times. The molecular weight of each polymer was over 30,000 , which was determined by gel permeation chromatography.

\section{Measurements}

JC dye dispersed polymer films were prepared as follows. A THF solution of JC dye was mixed well with a polymer THF solution. The concentration of the JC dye was $30 \mathrm{wt} \%$ of the matrix polymer. The polymer film was prepared on $\mathrm{SnO}_{2}$ coated glass electrode with spin-coating method. The film obtained was dried in vacuo at $25^{\circ} \mathrm{C}$ for $24 \mathrm{~h}$ to remove the solvent. All polymer films presented in this experiment were prepared with a film thickness of about $4 \mu \mathrm{m}$. An Ag electrode was subsequently attached to the polymer film as a counter electrode by spattering method (Sanyu Denshi, SC-701 quick coater), and a sandwich type cell was then constructed. The electrode area of the cell was $1.13 \mathrm{~cm}^{2}$.

The cell thus obtained was placed in a cryostat, and the pressure in the cryostat was reduced to under $10^{-5}$ Torr. Photoconductivity was measured with a $500 \mathrm{~W}$ Xe lamp (Ushio Denki UI-501C) and a dc power supply (Kikusui Denshi Model 103) at $30^{\circ} \mathrm{C}$. Light was irradiated from the $\mathrm{SnO}_{2}$ glass electrode side of the cell through a thermoabsorbent (IR cut off) filter. The energy of the irradiated light was estimated to be $256 \mathrm{~mW} \mathrm{~cm}^{-2}$ by a radiometer/photometer (EG \& G Model 550). The obtained signal was picked up with a probe connected to a vibrating reed electrometer (Advantest TR-84M), and recorded on an X-t recorder (Riken Denshi SP-H8V). Action spectral response of the photocurrent was measured at a constant electric field of $10^{4} \mathrm{~V} \mathrm{~cm}^{-1}$. Light from the Xe lamp was monochromatized through a single grating monochromator (Jovin Yvon H20 IR) using a suitable interference filter to remove undesir- 
able second and third interference wavelength light. In this measurement, photocurrent was evaluated as photo-induced current per incident photon unit.

UV-Vis absorption spectra of a JC dye/THF solution and a dispersed film were measured with a double beam spectrophotometer (Shimadzu UV-220).

Dc electrochemical behavior was evaluated from cyclic voltammograms measured with a potentiogalvanostat (Toho Technical Research Model 2020), a function generator (Hokuto Denko Model HB-111) and a Riken Denshi F-3DG recorder. For ac voltammetry, an oscillator (ac function generator, Kikusui Electronics model 459), a lock-in amplifier (NF Electronic Instruments Model LI-570) and an oscilloscope (Meguro Electronics MO1253) were connected to the apparatus for $\mathrm{dc}$ cyclic voltammetry. The details of the instrumentation for ac voltammetry were described previously. ${ }^{16}$ All electrochemical measurements were carried out at $20^{\circ} \mathrm{C}$.

\section{RESULTS AND DISCUSSION}

All the JC dyes consisted of electron donating part and electron accepting part bridged by an azo bond. JC-7 has structurally the same electron accepting part of JC-8 and stronger electron donating part than JC-8. On the other hand, JC-9 has the same electron donating part of JC-8 and stronger electron accepting part than JC-8. If intramolecular charge transfer occurs in these molecules through azo bonds, JC-7 and JC-9 have more electron donating and accepting characteristics than JC-8. The absorption spectrum for each JC dye in a THF dilute solution is shown in Figure 2. In general, the introduction of electron donating and accepting groups into a $\pi$ electron conjugated system makes the HOMO level increase and the LUMO level decrease. The formation of an intramolecular charge transfer complex, therefore, causes decrease in the energy gap between HOMO and LUMO, resulting in a red shift in the absorption spectrum of the molecules. In this case, although trans-type azo benzenes with no substituent showed an absorption peak at around $350 \mathrm{~nm}$ in a THF solution, each JC dye showed an absorption peak at a wavelength longer than $550 \mathrm{~nm}$. Each JC dye seemed to form an intramolecular charge transfer complex. An absorption peak for JC-7 or JC-9 in a THF solution was observed at a longer wavelength than that for JC-8. Molar extinction coefficients $\left(\varepsilon_{\max }\right)$ of JC-7, JC-8, JC-9 in a THF solution were $4.0 \times 10^{4}, 3.9 \times 10^{4}$, and $4.3 \times 10^{4}$ $\left(1 \mathrm{~mol}^{-1} \mathrm{~cm}^{-1}\right)$, respectively. These results

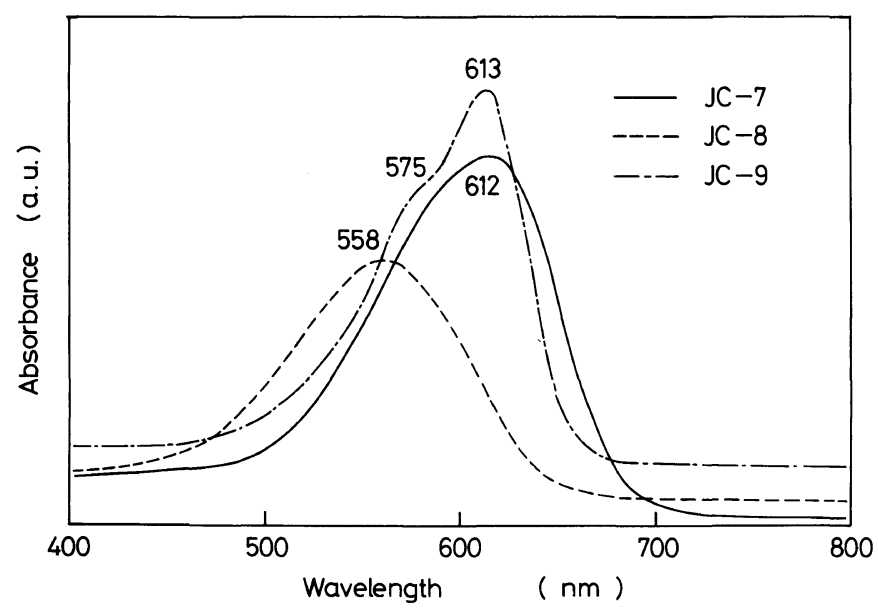

Figure 2. Absorption spectra for each JC dye in a THF solution. 
support the above consideration that JC-7 and JC-9 show more electron donating and accepting characteristics than JC-8.

Electric field $(E)$ dependence of the dark current and photocurrent was measured for a JC dye dispersed poly(carbonate) (PC) films, and a typical relationship for the JC-9 dispersed PC film is shown in Figure 3. In this figure, superscripts + and - for the dark current $\left(I_{\mathrm{d}}\right)$ and the photocurrent $\left(I_{\mathrm{p}}\right)$ are defined to be the polarity of $\mathrm{SnO}_{2}$ electrode at the irradiated side. Generally, the electric field dependence of the dark current for the cell is described by eq 1 .

$$
I=K \cdot E^{m}
$$

Where $I, K$, and $E$ are current, constant and electric field, respectively. This equation gives eq 2 in a common logarithmic form.

$$
\log I=m \cdot \log E+\log K
$$

The dark current in such a system is frequently associated with various effects such as

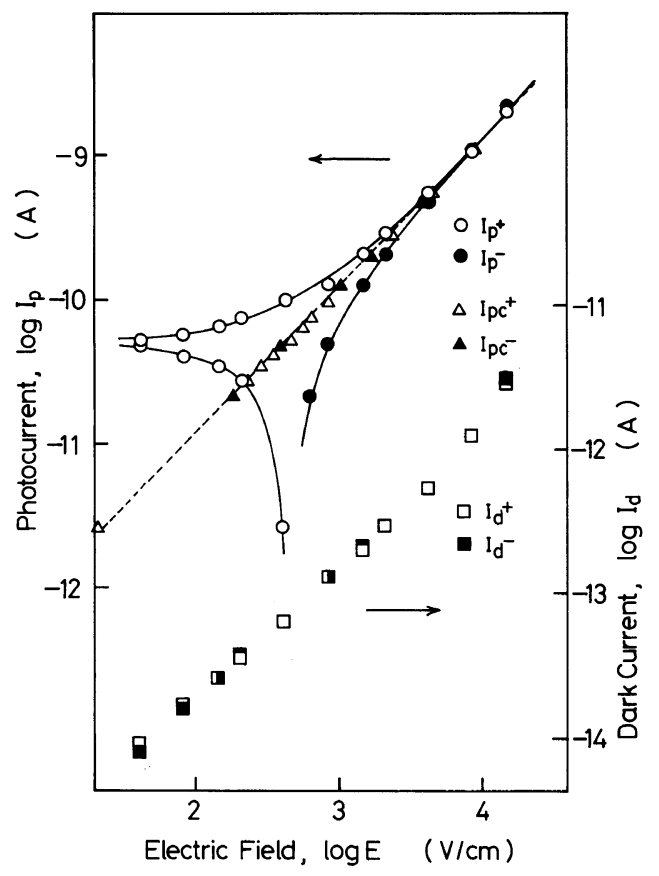

Figure 3. Electric field dependence of the dark current and photocurrent in each JC dye dispersed PC film.
Shottoky barrier, the Pool-Frenkel effect and so on. These effects can be estimated from the value of $m ; m=0.5$ for Shottoky barrier or Pool-Frenkel effect, $m=1$ for Ohmic behavior, and $m>1$ for space charge limited current. The relation between $\log I_{\mathrm{d}}$ and $\log E$ seemed to be linear and its slope was almost unity, suggesting that this cell is an ohmic system. The photocurrent for the JC-9/PC film was about $1.6 \times 10^{2}$ times larger than the dark current at the electric field of $1 \times 10^{4} \mathrm{~V} \mathrm{~cm}^{-1}$. A drop in the photocurrent was observed in an electric field of about $5 \times 10^{2} \mathrm{~V} \mathrm{~cm}^{-1}$ as shown in Figure 3. This was caused by the photoinduced voltage of the cell. The logarithmic correlation between the photocurrent and the electric field was corrected by removing the effect of photo-induced voltage. The correlation thus obtained was estimated to be linear, and its slope was almost unity, the same as the dark current. This suggest that the photocurrent is not affected by photo-induced space charge during irradiation. AC-2 and DO-2 were used to evaluate the effect of the in-

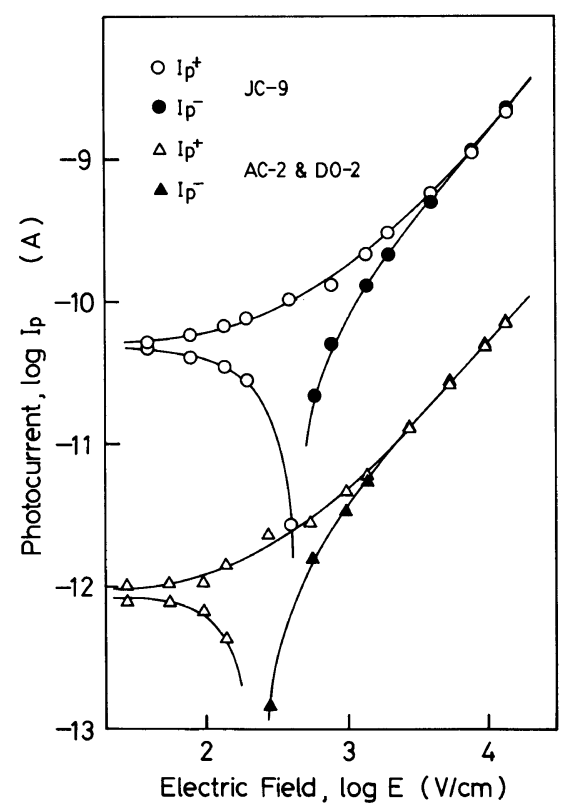

Figure 4. Electric field dependence of the photocurrent in JC-9 or AC-2 and DO-2 dispersed PC film. 
tramolecular charge transfer complex on the photocurrent in the PC dispersed system. Dark current for the AC-2 and DO-2 dispersed PC films was comparable to that for the JC-9 dispersed PC film. However, the photocurrent for the AC-2 and DO-2 dispersed PC film was about 30 times lower than that for the JC-9 dispersed PC film as shown in Figure 4.

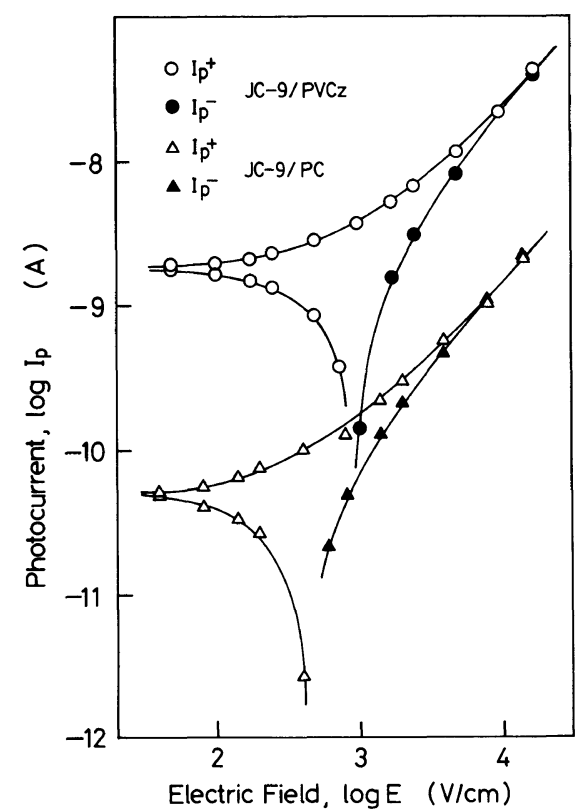

Figure 5. Typical relationship of photocurrent $v$ s. electric field for a JC-9 dispersed PVCz or PC film.
Furthermore, an azobenzene dispersed PC system showed a small photocurrent. These results suggest that intramolecular charge transfer in JC dye is effective for carrier generation and/or carrier transport.

Poly $(N$-vinyl carbazole) (PVCz) was used as a matrix polymer in order to prepare a system with superior photoconductive characteristics and to evaluate the effects of the polymer matrix on the photocurrent. $\mathrm{PVCz}$ is wellknown as a photoconductive polymer, and its characteristics are considerably enhanced when it is mixed with a suitable electron acceptor such as 2,4,7-trinitro-9-fluorenone (TNF). ${ }^{2,3}$ Electric field dependence of the dark current and the photocurrent were evaluated for JC dye dispersed $\mathrm{PVCz}$ films. A typical relationship of the photocurrent $v s$. electric field for a JC-9 dispersed PVCz film is shown in Figure 5, and compared with that for a PC system. There was little difference in the dark current between $\mathrm{PVCz}$ and $\mathrm{PC}$ systems. However, the photocurrent for the $\mathrm{PVCz}$ system was about one order larger than that for the PC ststem at the electric field of about $10^{4} \mathrm{~V} \mathrm{~cm}^{-1}$. PC is considered to be an inert matrix for photoconduction, but $\mathrm{PVCz}$ is a photoconductive matrix in itself. The characterisitcs of the polymer affect the photocurrent for each system. UV-Vis spectra for the JC-9

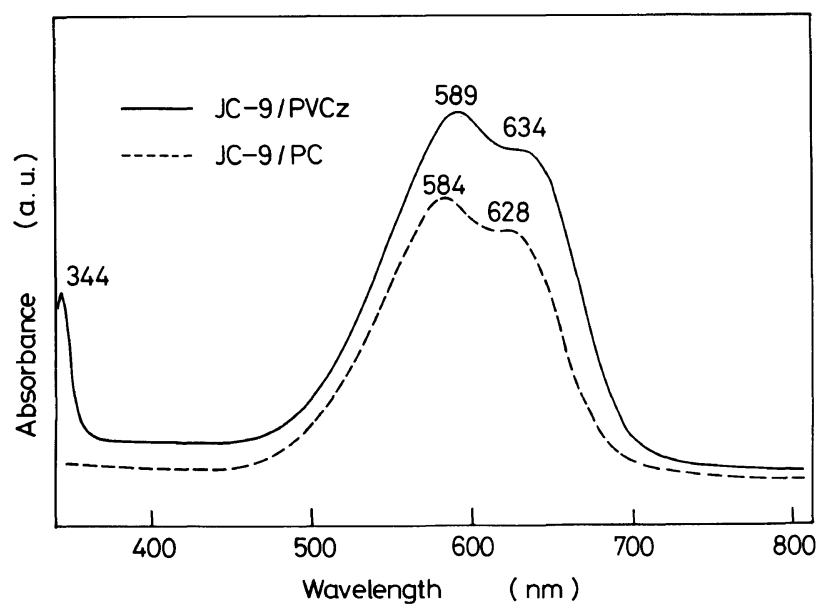

Figure 6. Absorption spectra for a JC-9 dispersed $\mathrm{PC}$ or $\mathrm{PVCz}$ film. 
dispersed PVCz and PC films were analyzed in order to evaluate the interaction between JC dye and polymer, and are summarized in Figure 6 . The absorption peak for JC-9 became broader and shifted to longer wavelength in a polymer matrix than in a THF solution as shown in Figure 2. This can be explained as due to a polymer effect. The absorption spectrum for JC-9 in a PVCz matrix was similar but shifted to a longer wavelength than that in the PC matrix. JC-9 was considered to have stronger electron accepting characteristics than others. JC-9 therefore interacted with PVCz in the film, resulting in a red shift of the absorption spectrum for the JC-9 dispersed PVCz film. Certainly, the photocurrent increment for the $\mathrm{PVCz}$ film is considerably affected by carrier generation and transport in the carbazolyl rings in PVCz. From the results of these absorption spectra, intermolecular interaction between $\mathrm{PVCz}$ and JC-9 may affect the photoconduction to some extent in this system.

Electrochemical analyses were conducted for each JC dye in order to evaluate the electron donating and accepting characteristics. Generally, half wave potentials of chemicals for oxidation $\left(E_{1 / 2(\text { ox })}\right)$ and reduction $\left(E_{1 / 2(\mathrm{red})}\right)$ have proportional correlation with ionization potential $(I P)$ and electron affinity $(E A)$, respectively. These correlations are expressed as follows:

$$
\begin{aligned}
& E_{1 / 2(\mathrm{ox})}=I P+E_{\mathrm{R}}^{+}-E_{\mathrm{R}}+\text { const. } \\
& E_{1 / 2 \text { (red) }}=E A+E_{\mathrm{R}}-E_{\mathrm{R}}^{-}+\text {const. }
\end{aligned}
$$

Table I. Half wave potential for oxidation and reduction of each $\mathrm{JC}$ dye

\begin{tabular}{cccc}
\hline \multirow{2}{*}{ Sample } & $\frac{E_{1 / 2(\mathrm{ox})}}{}$ & & $E_{1 / 2 \text { (red) }}$ \\
\cline { 2 - 2 } & $( \pm 0.02 \mathrm{~V})$ & & $( \pm 0.02 \mathrm{~V})$ \\
\hline JC-7 & +1.20 & & -0.47 \\
JC-8 & +1.32 & & -0.50 \\
JC-9 & +1.32 & & -0.42 \\
\hline
\end{tabular}

where $E_{\mathrm{R}}, E_{\mathrm{R}}^{+}$, and $E_{\mathrm{R}}^{-}$are solvation energy for neutral, cationic, and anionic molecules, respectively. Phase selective second harmonic ac polarography or voltammetry is considerably effective for determining the precise half wave potential for a system which contains systematic error due to the kinetic influence of the follow-up chemical reaction. ${ }^{17}$ Half wave potentials for oxidation and reduction of $\mathrm{JC}-7,8$, and 9 were measured in a THF solution with a phase selective second harmonic ac voltammetry, and are summarized in Table I. As DO-2 and AC-2 showed irreversible electrochemical reaction, we did not obtain $E_{1 / 2}$ for these molecules. Peak potentials for dc cyclic voltammetry, however, indicated that DO-2 and AC-2 had electron donating and accepting characteristics, respectively. In spite of the irreversible electrochemical reaction for DO-2 and AC-2, each JC dye as well as JC-9 showed reversible electrochemical reaction. This is evidence for intramolecular charge transfer in JC dye by bridging DO-2 and AC-2 through a $\pi$-electron conjugated azo bond. $E_{1 / 2(\mathrm{ox})}$ and $E_{1 / 2 \text { (red) }}$ represented electron elimination and injection properties for the chemicals, respectively. These were therefore effective to estimate the electron donating and accepting characteristics. In Table I, the absolute value of $E_{1 / 2 \text { (red) }}$ was smaller than that of $E_{1 / 2(\text { ox })}$ in each JC dye, suggesting that each $\mathrm{JC}$ dye itself had rather electron accepting characteristics than donating characteristics. $E_{1 / 2(\mathrm{ox})}$ of JC-8 was similar to that of JC-9. This was considered to be due to the same electron donating group for JC-8 and 9 . The small value of $E_{1 / 2(\text { ox })}$ for JC-7 indicated that $\mathrm{JC}-7$ had relatively stronger electron donating characteristics than JC-8 or JC-9. On the other hand, the absolute value of $E_{1 / 2 \text { (red) }}$ decreased in the sequence JC-8 $>$ JC$7>$ JC-9, suggesting that electron accepting characteristics increased in the order JC$8<$ JC- $7<$ JC-9.

The dark current $\left(I_{\mathrm{d}}\right)$ and the photocurrent $\left(I_{\mathrm{p}}{ }^{+}\right.$and $\left.I_{\mathrm{p}}{ }^{-}\right)$for each JC dye dispersed PVCz film at the electric field of $10^{4} \mathrm{~V} \mathrm{~cm}^{-1}$ are 
summarized in Table II. All of these values increased in the sequence JC-8 $<$ JC- $7<\mathrm{JC}-9$. This sequence was also maintained under an electric field ranging from $4 \times 10^{1}$ to $2 \times 10^{4}$ $\mathrm{V} \mathrm{cm}^{-1}$. Especially, in the JC-9 dispersed $\mathrm{PVCz}$ film, $2.1 \times 10^{3}$ times larger current was obtained by light irradiation. Electron accepting characteristics increased in the sequence JC- $8<$ JC- $7<$ JC-9 as mentioned in Table I. When $\mathrm{PVCz}$ was used as a polymer matrix, the photocurrent in the $\mathrm{JC}$ dye dispersed $\mathrm{PVCz}$ film was revealed to be considerably affected by the degree of electron accepting characteristics of JC dye. The same tendency was also noted for the dark current and photocurrent in the JC dye dispersed PC film in an electric field of $10^{4} \mathrm{~V} \mathrm{~cm}^{-1}$. In the PC system, the dark current and the photocurrent considerably depended on the electronic characteristics such as the degree of intramolecular charge transfer

Table II. Dark current and Photocurrent for each JC dye dispersed PVCz film

\begin{tabular}{ccccc}
\hline \multirow{2}{*}{ Sample } & \multicolumn{2}{c}{ Dark current } & & \multicolumn{2}{c}{ Photocurrent } \\
\cline { 2 - 3 } \cline { 4 - 5 } & $I_{\mathrm{d}} / \mathrm{A}$ & & $I_{\mathrm{p}^{+}} / \mathrm{A}$ & $I_{\mathrm{p}^{-}} / \mathrm{A}$ \\
\hline JC-7 & $8.9 \times 10^{-12}$ & & $6.9 \times 10^{-9}$ & $8.1 \times 10^{-9}$ \\
JC-8 & $8.4 \times 10^{-12}$ & & $5.6 \times 10^{-9}$ & $3.4 \times 10^{-9}$ \\
JC-9 & $1.1 \times 10^{-11}$ & & $2.3 \times 10^{-8}$ & $2.3 \times 10^{-8}$ \\
\hline
\end{tabular}

for JC dye itself because of the inert matrix of PC. Taking the data in Table I into account, the degree of intramolecular charge transfer was considered to increase in the sequence JC- $8<$ JC- $7<$ JC-9, and affected the photo carrier generation and/or transport in this sequence on the PC system.

The action spectrum of the photocurrent in JC-9 dispersed PVCz films is shown in Figure 7 together with its absorption spectrum. Irradiated light energy was about $50-80$ $\mu \mathrm{W} \mathrm{cm}^{-2}$ at each wavelength. In this figure, photocurrent is given as the corrected value per incident photon in unit area $\left(\mathrm{cm}^{2}\right)$. The action spectrum of each photocurrent $\left(I_{\mathrm{p}}{ }^{+}\right.$and $I_{\mathrm{p}}{ }^{-}$) showed antibatic correlation to the absorption spectrum. The same tendency was also found for other JC dye dispersed PVCz films. The irradiation light was considerably absorbed at the surface but not in the bulk of the film in the region corresponding to the absorption peak of the dye. Then, the carrier was generated and its density increased at the surface of the film. This probably brought about increase of the carrier recombination even in an electric field of $10^{4} \mathrm{~V} \mathrm{~cm}^{-1}$. Consequently, a decrease of photocurrent was observed in the region corresponding to the absorption peak of the JC dye. The peak of photocurrent at the longer wavelength lies in

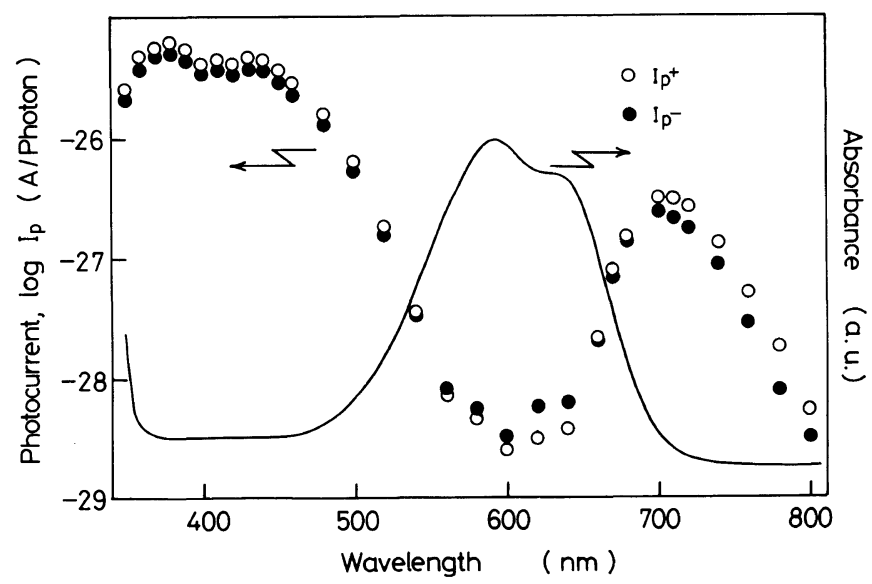

Figure 7. Action spectrum of photocurrent in a JC-9 dispersed $\mathrm{PVCz}$ film. 
the absorption edge for each JC dye in the $\mathrm{PVCz}$ film. This suggests that each dye is effective for carrier generation in this region. This also supports that carrier recombination is carried out in the region corresponding to the absorption peak of each JC dye. Namely, carrier generation took place in the $\mathrm{JC}$ dye at the absorption edge of the JC dye, and affected the photocurrent of the $\mathrm{PVCz}$ system. As shown in Figure 2, the absorption spectrum of JC-9 in $\mathrm{PVCz}$ shifted to a longer wavelength than that in the PC system. Intermolecular interaction between $\mathrm{JC}$ dye and $\mathrm{PVCz}$ was also considered to affect carrier generation to some extent in this region. Action spectral response of the photocurrent in the PC system, however, did not appear in our measurement with the same electric field and irradiated light energy as the PVCz system. This suggests that $\mathrm{JC}$ dye is effective for carrier generation rather than carrier transport, and carrier transport is assisted by the hopping through carbazole rings in the $\mathrm{PVCz}$ system. When light was irradiated from the $\mathrm{Ag}$ electrode side into the bulk of the film, antibatic correlation was also obtained between the photocurrent action spectrum and the absorption spectrum. Thus possibly the carrier is not generated at the interface of the electrode and the film sample but at the surface and/or in the bulk of the film.

\section{REFERENCES}

1. H. Hoegl, O. Sus, and W. Neugebauer, Ger. Patent 1068115 (1959) [Chem. Abstr., 55, 20742a (1961)].

2. R. M. Schafert, IBM Res. Dev., 15, 75 (1971).

3. W. D. Gill, J. Appl. Phys., 43, 5033 (1972).

4. G. Weiser, J. Appl. Phys., 43, 5028 (1972).

5. M. Yokoyama, S. Shimokihara, A. Matsubara, and H. Mikawa, J. Chem. Phys., 76, 724 (1982).

6. M. Yokoyama, K. Akiyama, N. Yamamori, H. Mikawa, and S. Kusabayashi, Polym. J., 17, 545 (1985)

7. H. W. Gibson, Polymer, 25, 3 (1984), reference cited there in

8. V. Gaidelis, V. Krisscinas, and E. Montrimas, Thin Solid Films, 38, 9 (1976).

9. T. Uryu, H. Ohkawa, and R. Oshima, Macromolecules, 20, 712 (1987).

10. C. J. Hu, R. Oshima, and M. Seno, J. Polym. Sci., Polym. Chem. Ed., 26, 1239 (1988).

11. H. Bassler, Phil. Mag., B, 50, 347 (1984).

12. M. Stolka, J. F. Yanus, and D. M. Pai, J. Phys. Chem., 88, 4707 (1984).

13. M. Sasaki, Nippon Kagaku Kaishi, 379 (1986).

14. R. Hirohashi and K. Kubo, Nippon Kagaku Kaishi, 373 (1986).

15. T. Tanaka and R. Hirohashi, Nippon Kagaku Kaishi, 867 (1989).

16. K. Akutagawa, N. Kobayashi, T. Tanaka, and R. Hirohashi, J. Soc. Photogr. Sci. Tech. Jpn., 52, 281 (1988).

17. A. M. Bond and D. E. Smith, Anal. Chem., 46, 1946 (1974). 